\title{
Research on Cooperative Scheduling Strategy for Twin-fab
}

\author{
Hao-Dan She \\ College of Electronics and Information Engineering, \\ Tongji University, \\ Shanghai, China \\ E-mail: 1433185@tongji.edu.cn
}

\author{
$\mathrm{Li} \mathrm{Li}$ \\ College of Electronics and Information Engineering, \\ Tongji University, \\ Shanghai, China \\ E-mail: lili@tongji.edu.cn
}

\begin{abstract}
This paper proposes a cooperative scheduling strategy for twin-fab model in semiconductor manufacturing, which consists of the strategy for job selecting equipment and the strategy for equipment selecting job. Dynamic bottleneck equipment, dynamic hurry-job and the workload balance between the two fabs are considered in the strategy for job selecting equipment, and the workload of successive equipment, the due date of jobs and the occupation time of jobs on equipment are considered in the strategy for equipment selecting job. Results in a twin-fab simulation model show that the proposed approach achieves improvement on the performance issues of throughput and on-time delivery rate.
\end{abstract}

Keywords-twin-fab; cooperative scheduling strategy; load balance

\section{INTRODUCTION}

The increasing demand on electronic products encourages the development of semiconductor manufacturing. The semiconductor companies need to expand their production scale to respond quickly to the changing in market demand. In order to decrease installation cost and increase production flexibility, the cooperative manufacturing style, such as the twin-fab, came into being. Twin-fab (shown in figure 1) means two independent and neighboring semiconductor production lines with similar process flow, are connected to each other by automatic transportation system (AMHS) so that a job can be transferred from one to another line to facilitate its smooth production. In twin-fab mode, these production lines can use the other's capacity for production so that the overall performance of twin-fab can be promoted.

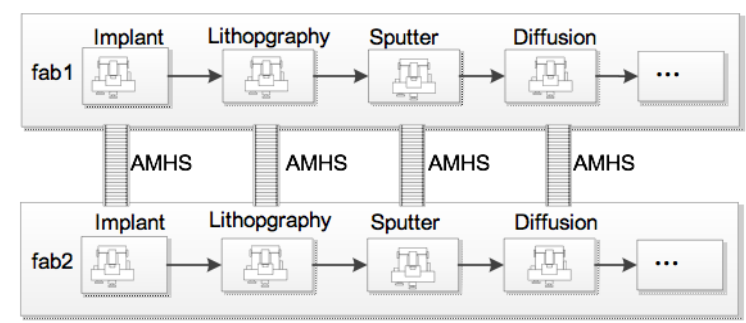

Figure 1. Cooperative manufacturing of twin-fab

Semiconductor manufacturing is one of the most complex manufacturing systems. The research on the scheduling of semiconductor production lines has always been a hot topic in the academic field. The scheduling process of a single semiconductor production line and a twin-fab (the addition parts comparing to the single fab enclosed in the grey rectangle) are shown in figure 2. 


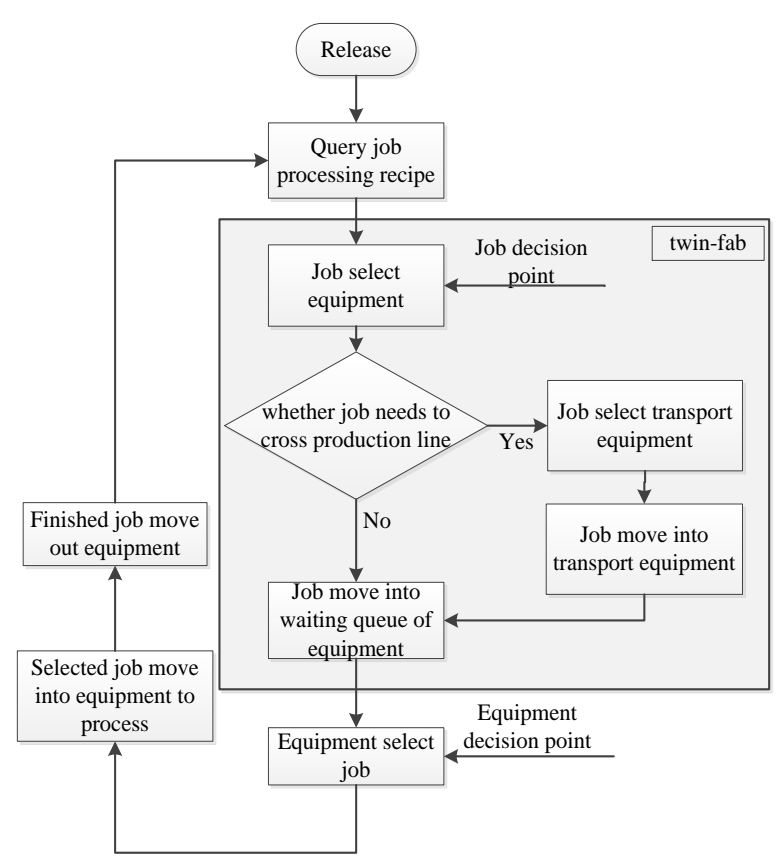

Figure 2. The cooperative scheduling process of twin-fab

The research on single semiconductor production line mainly focus on the strategy for equipment selecting job, which means an equipment makes decisions according to the real-time operating conditions at the decision-making point. For example, Wu et al. [1] proposed a scheduling strategy based on hybrid fuzzy logic, in which strategy the delivery time, the waiting time and the key characteristic parameters of jobs are considered; Chen et al. [2] proposed a method based on a mixed integer nonlinear programming model to select the optimal scheduling policy for the production line. Zhang et al. [3] proposed a dynamic bottleneck dispatching strategy, which uses a multi-level priority queuing model to divide three key parameters into different priority levels. The simulation results indicated that this dynamic dispatching method was superior to the traditional static scheduling methods.

As the appearance of cooperative manufacturing of twin-fab, the research on cooperative scheduling of twin-fab has gotten more and more attention. Comparing to the single semiconductor production line, twin-fab's scheduling problem is more complex.

Strategy for job selecting equipment, namely the job makes decisions on which equipment on which fab will be selected for its next step processing according to the realtime operating conditions at the job decision-making point. In the process of decision-making, the impact of the decision on the production line's performance should be considered, such as the workload balance of fabs and on-time delivery rate of the jobs. If the selected equipment is in another fab, the job should move into transport equipment to be transferred. Since the position, route and bottleneck transport equipment of AMHS will deeply affect the efficiency of the production line scheduling, the study of AMHS is another difficulty in the cooperative scheduling of twin-fab. For example, Liu et al. [4] studied the AMHS between parallel semiconductor production lines, which considered the AMHS transport bottlenecks, and the constraints of material handling caused by the plant layout, eventually the yield of parallel production line was improved. Chang et al. [5] considered the effect of job's transport time on scheduling and the bottleneck problem of AMHS, proposed that the workload balance between parallel lines should be considered and the frequency of cross line processing and meaningless transportation should be reduced.

By cooperative manufacturing, twin-fab can process multiple products in different orders simultaneously, make full use of production capacity, as well as the information flow between the two production lines and the different parts in the production lines, which help to improve production efficiency, optimize production scheduling. Currently there are few studies on cooperative scheduling of twin-fab, but they have lots of reference value. For example Liu et al.[6] considered both preventive maintenance and production planning in the study of cooperative manufacturing of twinfab; Chen et al.[7] systematic summarize cooperative manufacturing system of parallel production lines, model the system into four parts, including WIP-pulling module, workload accumulation module, wafer release time module and wafer start fab module, and they make different scheduling rules for each module, finally achieved the workload balanced of equipment; Tu et al. [8] proposed that in process of cooperative manufacturing, the number of products waiting before bottleneck equipment should be adjusted over time to avoid bottleneck equipment excessive blocking.

In this paper, cooperative manufacturing of twin-fab is studied, and a twin-fab cooperative scheduling strategy (TFCS) is proposed. TFCS considers a range of factors that influence the cooperative scheduling of twin-fab, and finally the strategy is verified by simulations.

\section{PROBLEM ASSUMPTIONS AND DEFINITIONS}

\section{A. Problem Assumptions}

During the study on cooperative scheduling of twin-fab, this paper makes the following assumptions:

- Two fabs in the twin-fab model are completely independent and have identical equipment;

- The transport equipment has enough capacity, i.e., there is no waiting time on transport equipment;

- The cross line transportation time of the jobs submits to the lognormal distribution of $(5,1)$;

- The transportation time of job in the same fab is not considered;

- The information related to dispatching is known, such as job process recipe, equipment function menu and equipment available time. These data can be obtained from MES or other automated systems.

\section{B. Definitions of Parameters And Variables}

Parameters and variables used in the TFCS strategy are defined in Table I. 
TABLE I. DEFINITIONS OF PARAMETERS AND VARIABLES

\begin{tabular}{|c|c|}
\hline Definition & Description \\
\hline 1 & Fab index \\
\hline $\mathrm{t} 1$ & Job decision point \\
\hline $\mathrm{t} 2$ & Equipment decision point \\
\hline $\mathrm{n}$ & $\mathrm{t} 2$ time waiting job index before $\mathrm{E}_{\mathrm{j}}$ \\
\hline \multirow[t]{2}{*}{$\mathrm{g}$} & t2 time job batch index \\
\hline & The ith decision job \\
\hline$E_{j}$ & $\begin{array}{l}\text { t1 time the ith equipment which can process } L_{j} \text { 's } \\
\text { next step }\end{array}$ \\
\hline$Q_{i}$ & t1 time the queue number of $\mathrm{L}_{\mathrm{i}}$ \\
\hline$W L_{j}$ & t1 time $E_{j}$ 's workload \\
\hline$P_{j}^{n}$ & The processing time of lot $n$ on $E_{j}$ \\
\hline$T_{j}$ & The available time of $E_{j}$ \\
\hline$E_{k}$ & The kth decision equipment \\
\hline$N L_{j}$ & $\begin{array}{l}\text { t1 time the workload of location where process } \mathrm{L}_{\mathrm{i}} \\
\text { next two step }\end{array}$ \\
\hline$N_{j}^{1}$ & $\begin{array}{l}\text { t1 time the number of available equipment of } \\
\text { location } E_{j} \text { belongs }\end{array}$ \\
\hline$N_{j}^{2}$ & $\begin{array}{l}\text { t1 time the number of available equipment of } \\
\text { location where will process } L_{i} \text { next two step }\end{array}$ \\
\hline$w_{l}$ & t1 time fab l's workload balance ratio \\
\hline$W_{l}$ & t1 time the number of WIP on fab 1 \\
\hline $\mathrm{D}$ & The fix number of WIP \\
\hline$m_{l}$ & t1 time fab l's MOV ration \\
\hline$M_{l}$ & fab l's daily MOV of last day \\
\hline $\bar{M}$ & Two fabs' mean daily MOV of last day \\
\hline$\Gamma_{i}^{j}$ & $\mathrm{t} 1$ time $\mathrm{E}_{\mathrm{j}}$ 's priority \\
\hline$F_{j}$ & $\mathrm{Li}$ and $\mathrm{E}_{\mathrm{j}}$ on the same fab \\
\hline$L_{j}$ & Location where $\mathrm{E}_{\mathrm{j}}$ belongs \\
\hline$L L_{j}$ & $\mathrm{t} 1$ time the workload of location $\mathrm{j}$ \\
\hline$D_{k}^{h}$ & Due date of job h \\
\hline$O_{k}^{h}$ & Ratio of $\mathrm{CT}$ and processing time of $\mathrm{L}_{\mathrm{h}}$ \\
\hline$Q_{k}^{h}$ & The occupation time of $\mathrm{L}_{\mathrm{h}}$ on $\mathrm{E}_{\mathrm{k}}$ \\
\hline$M_{k}$ & $\mathrm{t} 2$ time the number of job waiting before $\mathrm{E}_{\mathrm{k}}$ \\
\hline$P_{k}^{g}$ & The processing time of batch $g$ on $E_{k}$ \\
\hline$\overline{N L_{k}^{g}}$ & t2 time mean downstream workload of batch $g$ \\
\hline$x_{i}$ & Whether $L_{i}$ will finish \\
\hline$\tau_{k}^{h}$ & t2 time the priority of $L_{h}$ waiting before $E_{k}$ \\
\hline$x_{k}^{h}$ & Whether the hth job before $E_{k}$ will finish \\
\hline$i F_{k}^{h}$ & Is finish variables \\
\hline$R_{k}^{h}$ & t2 time $\mathrm{L}_{\mathrm{h}}$ 's hurry degree \\
\hline$G_{k}^{g}$ & The priority of batch $g$ \\
\hline$B_{k}^{g}$ & The batch size of batch $g$ on $E_{k}$ \\
\hline$H_{k}^{g}$ & The number of hurry-job in batch $g$ before $E_{k}$ \\
\hline
\end{tabular}

III. METHOD DESCRIPTION
In order to achieve the cooperative manufacturing of twin-fab, this paper firstly proposes a job selecting equipment strategy named Earliest Process strategy (EP), and on basis of this, the TFCS strategy is put forward.

\section{A. EP strategy}

In twin-fab, equipments of the same type use exactly the same function recipe. When a job selects equipment for its next step, it can choose any production line. In this way cooperative manufacturing is realized.

EP strategy mainly considers the queuing number of job before the equipment. Its purpose is that the decision job can be processed as early as possible. The decision flow is as follows.

- $\quad$ Step1. Firstly decision job $\mathrm{L}_{\mathrm{i}}$ queries the equipment groups on two fabs which can process its next step according to process recipe, then get equipment group $\sum E_{j}$;

- Step2. For every equipment $E_{j}$ in group $\sum E_{j}$, sorting the waiting jobs by waiting time in descending order;

- Step3. Query and save the waiting sequence $Q_{i}$ of decision job $\mathrm{L}_{\mathrm{i}}$ for equipment of group $\sum E_{j}$;

- Step4.Sorting group $\sum E_{j}$ by $Q_{i}$ in ascending order, which means where the decision job's sequence is more near the front, the higher priority the equipment gets to process job's next step;

- Step5. Selecting the equipment has smallest $Q_{i}$ for $\mathrm{L}_{\mathrm{i}}$ 's next step.

In this strategy, jobs can be transferred from one fab to another, so as to realize two fabs' cooperative manufacturing. However, this strategy only considers the condition that jobs could be processed as early as possible, there are many other factors that will affect the performance of fabs, such as the workload balance of fabs or locations and the move speed of jobs on fabs, so the EP strategy is deserved to be improved.

\section{B. TFCS Strategy}

The TFCS strategy comprehensive considers various influence factors. This strategy consists two parts. The first part is twin-fab selecting equipment strategy (TFSE), the factors this part mainly considered are dynamic bottleneck equipment, dynamic hurry-jobs and the workload balance of fabs; the second part is twin-fab selecting job strategy (TFSJ), the factors this part mainly considered are the workload of successive equipment, the due date of jobs and the occupation time of jobs on equipment. This paper uses both TFSE strategy and TFSJ strategy to keep the workload balance of the whole fabs and locations inside, at the same time improve the overall performance of twin-fab.

\section{1) TFSE strategy}

TFSE strategy is a strategy jobs used to make decisions at job decision-point. The purpose is selecting the proper equipment for its next step. This strategy mainly considers following factors.

a) Equipment related: Firstly $\mathrm{Li}$ queries the equipment groups in two lines which can process its next 
step according to process recipe, then get equipment group $\sum E_{j}$. For every $E_{j}$ in $\sum E_{j}$, calculate its workload $W L_{j}$, the calculation is as formula (1).

$$
W L_{j}=\frac{\sum P_{j}^{n}}{T_{j}}
$$

Formula (1) means at the job decision-point, the more occupation time of waiting jobs on equipment, the heavier workload equipment gets. In order to avoid equipment bottlenecks and speed up the movement of jobs, the equipment with lower workload should be given high priority.

b) Location related: For every $E_{j}$ in $\sum E_{j}$, calculate the location workload $L L_{j}$ of location $E_{j}$ belongs, and then calculate the location workload $N L_{j}$ of location which will process job's next two steps on line $E_{j}$ belongs. The calculation is as shown in the formula (2).

$$
\begin{aligned}
L L_{j} & =\frac{\sum W L_{m}^{1}}{N_{j}^{1}} \\
N L_{j} & =\frac{\sum W L_{m}^{2}}{N_{j}^{2}}
\end{aligned}
$$

Formula (2) means at the job decision-point, the lower workload of locations, the faster the job will move, which can decrease the frequency of unnecessary cross fab processing. So the lower workload of locations, the higher priority the available equipment will get.

c) Fab related: In order to keep the WIP balance of these two fabs, avoid one fab blocking while another fab idle, this strategy needs to consider the WIP balance ratio of fabs. The WIP balance ratio $w_{l}$ of fab 1 can be calculated as formula (3).

$$
w_{l}=\frac{W_{l}-D}{D}
$$

In the formula (3), the greater $w_{l}$ means the more WIP on this fab. Since the total number of WIP is certain, jobs should be moved to the fab that has fewer WIP to maintain the WIP balance of fabs, which means the fab has smaller $w_{l}$ gets higher priority.

d) Fab related: In order to process job faster, this strategy considers the performance index MOV, which indicates the process rapid of fab, the greater value of MOV means the fab process job faster. In order to realize real-time dispatching, this strategy considers the last daily MOV value of fab $E_{j}$ belongs. The movement ratio $m_{t}$ can be calculated by formula (4).

$$
m_{l}=\frac{\bar{M}-M_{l}}{\bar{M}}
$$

Formula (4) means at the job decision-point, the fab has smaller value of ${ }^{m_{l}}$ gets the higher priority.

e) Special case: When job will finish all steps on the recipe after next step, and enter into finished location, then the fab where this product be released has higher priority, which as shown in formula (5).

$$
\text { if } \mathrm{x}_{i}=1 \text {, then } F_{j}=M I N \text {. }
$$

The purpose of formula (5) is to classify the products according to order, and move the product to the right fab as soon as possible. The TFSE strategy comprehensive considers above five factors which will affect the performance of twin-fab, calculate the priority of each available equipment $E_{j}$ as formula (6), the equipment has the highest priority will be selected to process the decision job's next step.

$$
\Gamma_{i}^{j}\left(\mathrm{t}_{1}\right)=-\left(W L_{j}+B L_{j}+N L_{j}+w_{l}+m_{l}+F_{j}\right)
$$

If the selected equipment is not on the same fab with decision job, then the decision job need to be moved into transport equipment first and then be transferred to the selected equipment on the other fab.

\section{2) TFSJ Strategy}

When equipment becomes available, it needs to select the next batch jobs to process from the waiting queue before equipment. Due to the different processing condition of jobs waiting before equipment, the decision equipment needs to consider variety of influence factors to calculate waiting jobs' priority. The TFSJ strategy can be divided into nonbatch processing equipment selecting job strategy and batch processing equipment selecting job strategy.

a) Non-batch processing equipment selecting job strategy

For these non-batch processing equipment, this strategy mainly considers the equipment's dynamic bottleneck, which means at the decision-point, the equipment firstly calculates its workload level, and then according to whether it is bottleneck equipment and whether it has hurry-jobs in waiting queue chooses different strategy for scheduling. The pseudo code of this part is shown in Figure 3.

After waiting before decision equipment, the decision equipment will select the job has the highest priority to process. 


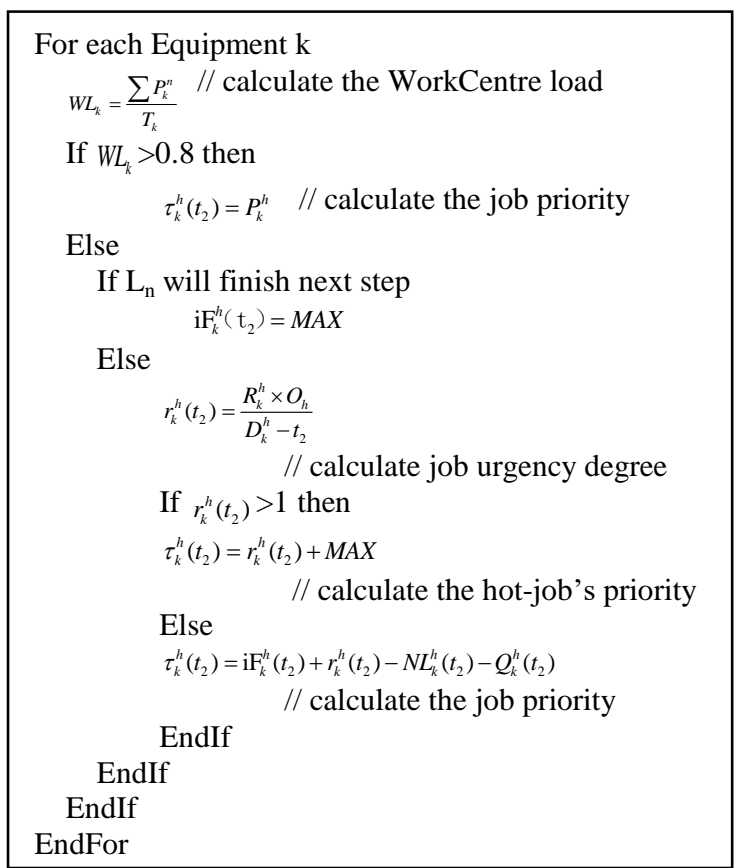

Figure 3. The pseudo code of TFSJ method(Non-batching equipment)

$$
\begin{aligned}
& \text { For each parallel equipment } \mathrm{k} \text { grouping job by recipe } \\
& \tau_{k}^{h}\left(t_{2}\right)=\mathrm{iF}_{k}^{h}\left(t_{2}\right)+r_{k}^{h}\left(t_{2}\right)-N L_{k}^{h}\left(t_{2}\right)-Q_{k}^{h}\left(t_{2}\right) \\
& \text { // calculate the job priority } \\
& \left.\begin{array}{l}
W L_{k}=\frac{M_{k}}{M N} \\
M N=\frac{T_{k}}{\min \left(P_{g}\right)}
\end{array}\right\} / / \text { calculate the WorkCentre load } \\
& G_{k}^{g}\left(t_{2}\right)=\frac{B_{g}}{\max \left(B_{g}\right)}-\frac{P_{g}}{\max \left(P_{g}\right)} \\
& \text { // calculate the batch } \mathrm{g} \text { priority } \\
& r_{k}^{h}\left(t_{2}\right)=\frac{R_{k}^{h} \times O_{h}}{D_{k}^{h}-t_{2}} \quad / / \text { calculate job urgency degree } \\
& \text { If exist } r_{k}^{h}\left(t_{2}\right)>1 \text { then } \\
& G_{k}^{g}\left(t_{2}\right)=\frac{B_{k}^{g}}{\max \left(B_{k}^{g}\right)}-\frac{P_{k}^{g}}{\max \left(P_{k}^{g}\right)}+\frac{H_{k}^{g}}{B_{k}^{g}} \\
& \text { // calculate the batch priority } \\
& G_{k}^{g}\left(t_{2}\right)=\frac{B_{k}^{g}}{\max \left(B_{k}^{g}\right)}-\frac{P_{k}^{g}}{\max \left(P_{k}^{g}\right)}+\frac{H_{k}^{g}}{B_{k}^{g}}-\frac{\overline{N L_{k}^{g}}}{\max \left(\overline{N L_{k}^{g}}\right)} \\
& \text { EndIf } \\
& \text { EndIf } \\
& \text { EndFor } \\
& \text { EndFor }
\end{aligned}
$$

Figure 4. The pseudo code of TFSJ method(Batching equipment) b) Batch processing equipment selecting job strategy

For batch processing equipment, this strategy considers the dynamic bottleneck of equipment, the proportion of finishing lot in the batch, the size of batch and the occupation time on the equipment. The pseudo code of this part is shown in Figure 4.

After complete calculating the priority of every batch waiting before decision equipment, the decision equipment will select the batch that has the highest priority to process.

\section{SIMULATION VERIFICATION AND RESULT ANALYSIS}

This paper takes a large number of historical data of 6 inch wafer production line in a semiconductor manufacturing enterprises of Shanghai as foundation, according to the actual need of enterprise, combining with dynamic modeling method, using the software of SIMENS Tecnomatix Plant Simulation to build a twin-fab simulation model, which keeps consistent with the life, and based on this simulation model conducts a series of experiments.

The simulation model consists of two independent semiconductor fab, every fab has nine processing locations, and separately has five kinds of products to keep different processing condition. In addition, there are five transport equipment in each fab used to transfer job from one fab to another. The product order is provided by the historical data of the semiconductor enterprise.

This paper conducts simulation experiments using different job selecting equipment strategy and equipment selecting job strategy combinations, then running ninety simulation days to conduct simulation verification. In these experiments, the release mode is all constant work in process (CONWIP) mode.

In order to verify the superiority of cooperative manufacturing, this paper conducts a set of comparison experiments that two semiconductor fab process jobs independently. Case 1 means two independent semiconductor fab, jobs can only process on one fab, the job selecting equipment strategy is FIFO, which means the nearest equipment has the highest priority to be selected; the equipment selecting job strategy is FIFO, which means the job first enter into equipment has the highest priority to be selected.

Other experiments are based on cooperative manufacturing of twin-fab, which means two semiconductor fabs can cooperative manufacture, jobs can be transferred to another fab to be processed, and the specific strategy is as table 2 shows.

TABLE II. STRATRGY OF DIFFERENT CASES

\begin{tabular}{|c|c|c|}
\hline Case & $\begin{array}{c}\text { Equipment selecting } \\
\text { job strategy }\end{array}$ & $\begin{array}{c}\text { Job selecting } \\
\text { equipment strategy }\end{array}$ \\
\hline Case2 & EP & FIFO \\
\hline Case3 & TFSJ & FIFO \\
\hline Case4 & EP & TFSE \\
\hline Case5 & TFSJ & TFSE \\
\hline
\end{tabular}


In these simulation experiments, each strategy combination has six processing conditions, which means each fab has different WIP when releasing, the number of WIP separately is 2500/5000/6250/7500/10000. Then performances as the total throughput $\mathrm{TP}$, the average cycle time CT and the on time delivery rate ODR are statistics from simulation data to compare the effect of different strategy combinations on twin-fab simulation model.

\section{1) Total throughput TP performance compare}

The result of total throughput is as Figure 5 shown.

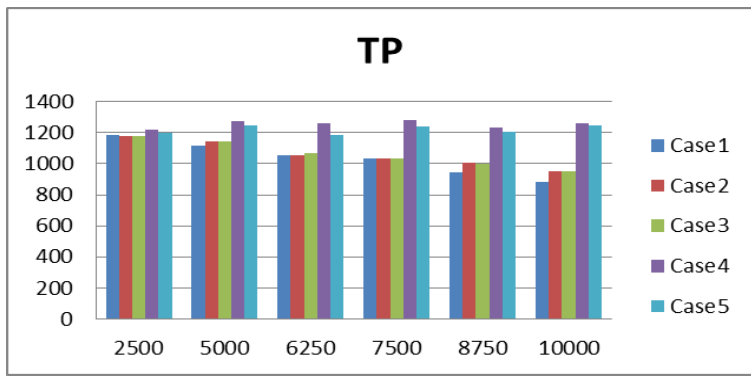

Figure 5. Total throughput performance comparison

From figure 5, this paper concludes as follows:

- $\quad$ Firstly, If only improve the job selecting equipment strategy, and the equipment selecting job strategy is FIFO, when the number of WIP are 2500,5000,6250 and 7500 , the throughput is basically flat. However, when the number of WIP is greater, the advantage of cooperative manufacturing of twin-fab becomes obvious. Comparing to case 1 , when the number of WIP is 8750 , case 2 and case 3 separately improve $6.8 \%$ and $6.1 \%$; when the number of WIP is 10000 , case 2 and case 3 separately improve $7.5 \%$ and $7.8 \%$. Therefore, the more the beginning WIP released, the effect of cooperative manufacturing of twin-fab the better, this is exactly what real production lines needs.

- Secondly, when improve both job selecting equipment strategy and equipment selecting job strategy, the throughput under any process condition gets great improved, especially when the number of WIP is 10000 , comparing to case 1 , case 4 and case 5 separately improve $42 \%$ and $41 \%$. The main reason is that no matter job selecting equipment strategy or equipment selecting job strategy, they all mean to accelerate the job's processing speed, so that the job could be completed as soon as possible.

2) The average cycle time result compare:

The result of the average cycle time is as figure 6 shown.

The analysis shows that when the number of WIP are 2500, 5000 and 6250, the job's average cycle time under five cases are similar, when the number of WIP is 7500,8750 and 10000 , due to the improvement of equipment selecting job strategy, the job's average cycle time is greatly shortened. When the number of WIP is
10000, comparing to case 1 , case 4 and case 5 separately shorten $18.8 \%$ and $16.4 \%$. Therefore, when the workload of fabs is heavy, the improvement of equipment selecting job strategy has a great influence on the performance of twin-fab.

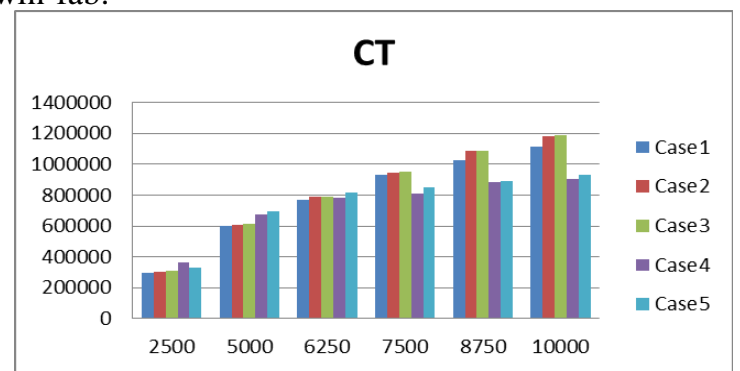

Figure 6 . The average cycle time performance comparison

3) The on time delivery rate result compare

The result of the on time delivery rate is as figure 7 shown.

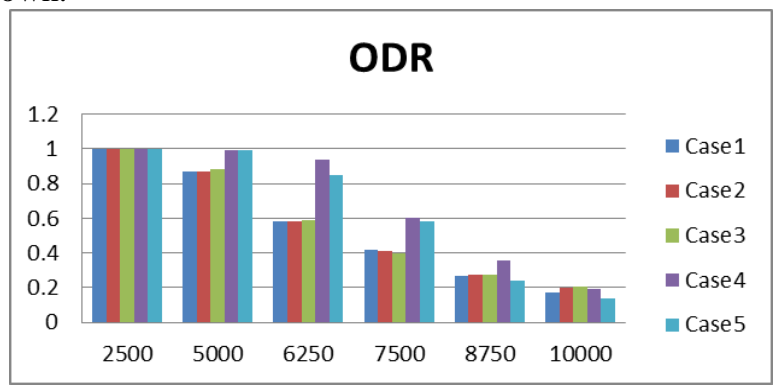

Figure 7. The on time delivery rate performance comparison

The analysis shows that when these two fabs cooperative manufacturing, if the equipment selecting job strategy gets improvement, the ODR performance will be improved deeply. This is because the condition that the hurry-jobs and finishing job gets highest priority is considered in equipment selecting job strategy.

\section{CONCLUSION}

This paper is based on the actual need of semiconductor manufacturing enterprise, combined with the characteristics of scheduling, studies the cooperative manufacturing of twinfab. This paper analysis the difference of scheduling between single semiconductor production line and twin-fab, and in order to realize cooperative scheduling of twin-fab, the TFCS strategy is proposed, which strategy is scheduling according to real time process condition of production line.

In order to verify TFCS strategy's validation, a series of simulation experiments is conducted. Results show that the cooperative manufacturing of twin-fab can deeply improve the total throughput, the average cycle time and the on time delivery rate of fabs.

On the other hand, the TFCS strategy this paper proposed can be further optimized, such as give different weight to these influence factors considered in strategy, and according to different requirements of fabs' performances, calculate 
different weight combinations, so as to the performance of fabs gets targeted improvement.

\section{ACKNOWLEDGMENT}

This paper is supported in part by National Natural Science Foundation of China under grants No.51475334.

\section{REFERENCES}

[1] Lihui Wu, Yinbin Sun, Gong Zhang, and Jie Zhang, "A fuzzy logicbased and hybrid dispatching policy for interbay material handling system in $300 \mathrm{~mm}$ semiconductor manufacturing system," Proceedings 8th International Conference on Supply Chain Management and Information Systems (SCMIS 2010), pp66-70, 2010.

[2] Chin-Feng Chen, Kun-Jia Wu , Chuei-Tin Chang, "Generation and verification of optimal dispatching policies for multi-product multitool semiconductor manufacturing processes," Computers \& Chemical Engineering, v 52, p 112-21, 10 May 2013.

[3] Zhang Huai, Jiang Zhibin, and Lee Yen-Fei, An approach of dynamic bottleneck machine dispatching for semiconductor wafer fab, 2007
International Symposium on Semiconductor Manufacturing, p 253-6, 2007.

[4] Alex LiuKuo, Chia-Cheng, Chien-Chih Chiu. Efficiency AMHS For Twin FAB Manufacture. Semiconductor Manufacturing. 2007:1-5.

[5] Cheng-Sheng Chang, Hsi-Lo Lo, Cheng-Chung Pan, De-Lung Wu. Advanced Effective Dispatching Method for $300 \mathrm{~mm}$ Twin-Phase Twin-Fab. Advanced Semiconductor Manufacturing Conference, 2009.ASMC '09. IEEE/SEMI. IEEE. 2009:76-79.

[6] Liu Qing, Li Hua. Scheduling problems with preventive maintenance on two parallel production lines. Management and Service Science (MASS), 2010 International Conference on IEEE. 2010:1 - 4.

[7] James C. Chen, Yang-Chih Fan, Chia-Wen Chen. Capacity requirements planning for twin Fabs of wafer fabrication. International Journal of Production Research. 2009,8,47(16): 44734496.

[8] Tu YingMei, C.W. Lu, S.H. Chang. Model to Evaluate Production Performance of Twin-Fab under Capacity Support. Advanced Materials Research. 2013(694-697):3453-3457. 